\title{
Infrared thermography as a tool to elaborate procedures for predictive maintenance of ball mills equipment
}

\author{
by R. Władysiak*, T. Pacyniak* \\ * Lodz University of Technology, Department of Materials Engineering and Production Systems, \\ 1/15 Stefanowskiego Str., 90-924 Lodz, Poland, ryszard.wladysiak@p.lodz.pl
}

\begin{abstract}
This paper describes the application of infrared thermography for work conditions monitoring of ball mills that are main machines of ceramic products manufacturing process. The thermal results was recorded using the thermal imaging camera. The tests on the engines were carried a couple times, and the measurement area was narrowed to verify the couplings and bearings of the machines. The work demonstrates that the use of thermal imaging camera allows to elaborate their maintenance's procedures.
\end{abstract}

\section{Introduction}

Infrared thermography (IRT) has many advantages over other technologies [1-4]. IRT is a non-contact technology, the devices used are not in contact with the source of heat. In this way, the temperature of extremely hot objects or dangerous products, such as acids, can be measured safely, keeping the user out of danger. IRT provides twodimensional thermal images, which make a comparison between areas of the target possible. IRT is in real time, which enables not only high-speed scanning of stationary targets, but also acquisition from fast-moving targets and from fast-changing thermal patterns. IRT has none of the harmful radiation effects of technologies, such as X-ray imaging. Thus, it is suitable for prolonged and repeated use and it is a non-invasive technique.

In most electrical systems, a stable temperature over time is important to avoid system breakdowns. Sudden hot spots can indicate faulty areas and connections, e.g. in electric circuits and heating systems. It would obviously be of great value if devices that are starting to overheat could be detected before they breakdown. One of the reasons for using thermal imaging for temperature measurement is that it is not in contact with the target. Thermal imaging can be applied as a diagnostic tool for electrical joints in power transmission systems [5], and for automatic detection of the thermal conditions of other electrical installations [6]. The thermographic studies conducted by the author's team show that the thermal imaging camera enables the analysis of the metal and alloy crystallisation process [7] as well as the diagnosis of foundry defects and monitoring of electrical devices [8].

The new approach to the tasks of the maintenance department allows to move away from prevention towards predictive maintenance [9]. This standard is intended to minimize, and consequently even to exclude downtime, at the expense of increased efficiency. The main tools of this approach are continuous monitoring of performance parameters, testing with visual inspection using non-invasive techniques.

\section{Work methodology, experiments}

The subject of the research was the ball mill drives (Fig. 1), which are the key machines of manufacturing systems in the production of ceramic products. This means that their operation should not be interrupted by unplanned stops or reduced performance. The total weight of the mill along with raw materials and alubit balls is over 50 tons.

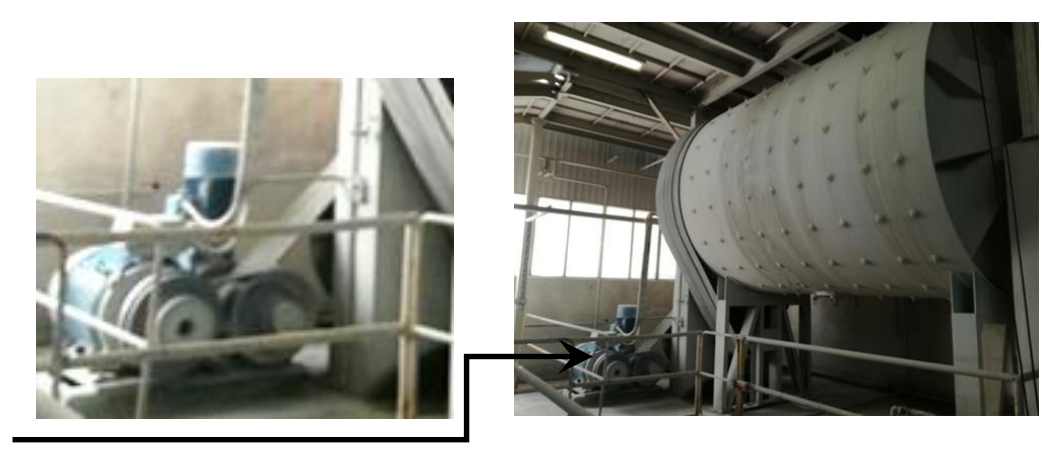

Fig.1. Production station of research with electric drive 
The mills are three-phase electric generators with a capacity of approximately $110 \mathrm{~kW}$. Investigations were carried out on cast iron engine housing of the engine and hydrodynamic coupling. During work these components achieve higher temperatures than other elements and are a frequent cause of mills' failure.

The tests of electric drives were carried out using the FLIR E40bx thermal imaging camera. This camera is equipped with an uncooled sensor detector with a resolution of $160 \times 120$ pixels and a thermal sensitivity $45 \mathrm{mK}$. The object's emissivity was verified by contact measurement using a thermocouple as a temperature sensor. Monitoring was carried out using the method of thermal trend analysis. The tests were carried out at intervals of several to several days. The final stage is a comparison of images and temperature distributions of the intended areas of the device. Obtained as a consequence of research, knowledge about deviations of temperature from nominal values and the frequency of their occurrence aims at eliminating possible failure of production stations.

In order to perform correct thermography tests, a test procedure was developed that defined the principles of measurements. It contained the required order of performed and the conditions of the activities performed. The commencement of the measurement at the production site requires the evaluation of safety hazards at the testing site and obtaining the consent of the occupational health and safety inspector for staying in the danger zone.

Further work focused on ensuring the availability of observations of the facility and preparing it for measurement, ensuring nominal load, disassembly of safety guards (Fig. 2) and identification of environmental conditions. Efforts should also be made to minimize the influence of external factors on the examined object, such as solar radiation, location of the observation point and ensuring the appropriate field of view of the object and determining the geometrical properties of the object.
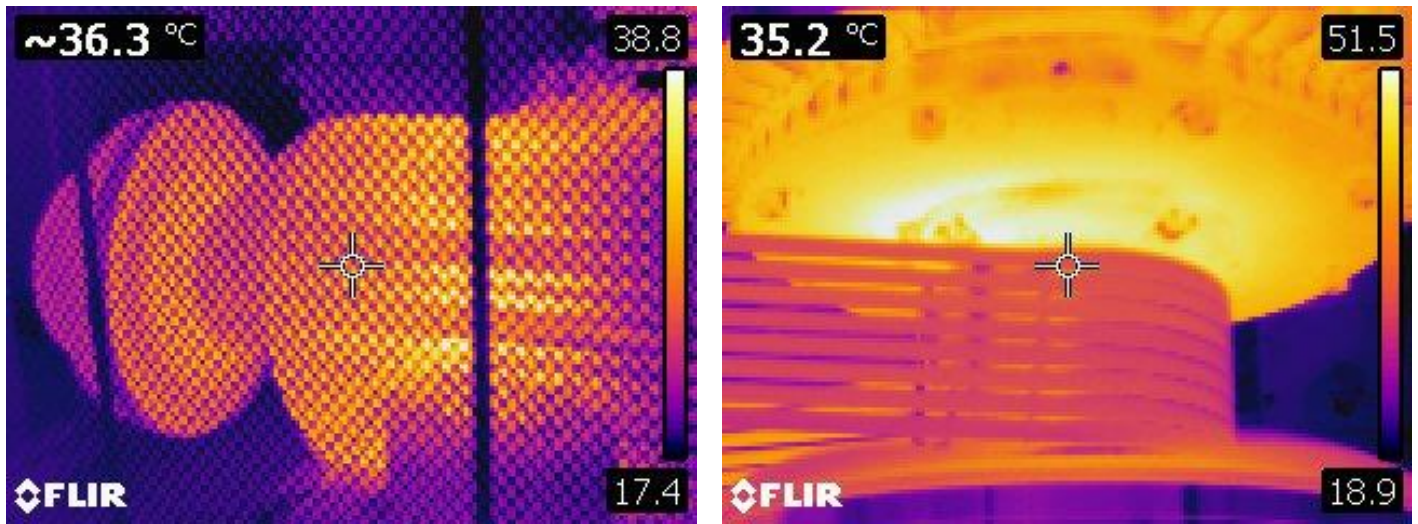

Fig. 2. Time thermogram of the electric motor and open belt drive observed by and without the protective cover

In order to accurately determine the required parameters, the test procedure includes a checklist. It requires the operator to enter parameters of the tested object and environmental conditions such as working time, load, emissivity, distance from the camera, ambient temperature, humidity, dustiness. Measurements of the selected object should be made in almost identical conditions defined by the distance between the camera and the object, the angle of observation, the size of the area of the analyzed surface and the lighting of the object.

\section{Research results and analysis}

Figures 3 and 4 present the test results of the drives of two ball mills. The results of the tests are accompanied by images of devices in visible light to improve the analysis of the studied areas.
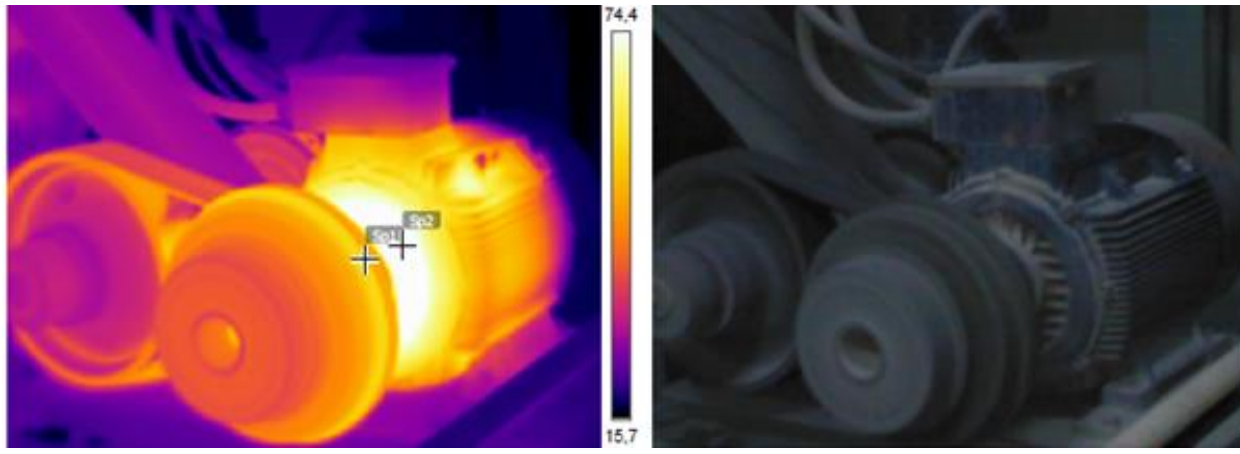

Fig.3. Thermogram and photo of ball mill No 1 

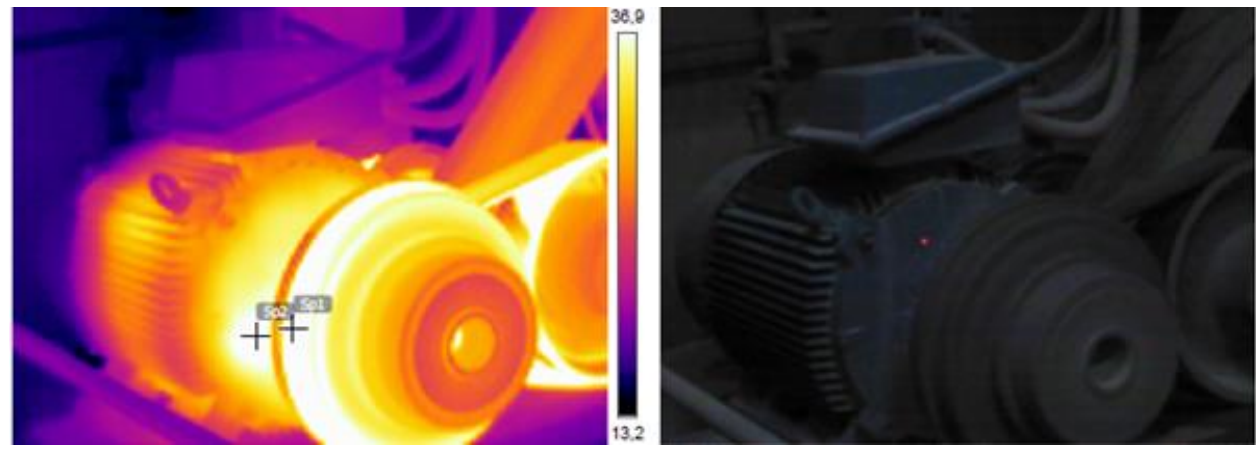

Fig. 4. Thermogram and photo of ball mill No 2

As a result of the preliminary tests carried out, the measurement area was narrowed down to verify the coupling at point Sp 1 and bearings - Sp 2. The research shows that selected areas heat up to the most in the whole device.

Figures 5-8 present the development of temperature test results (adequately $\mathrm{Sp} 1, \mathrm{Sp} 2$ ) for a representative ball mill drive obtained during various working shifts. The research shows that during the continuous operation of the mill the temperature of the motor in the bearing area increases for the first $3.5 \mathrm{~h}$ in an asymptotic manner in the range from about $45 \mathrm{C}$ to $90 \mathrm{C}$. In the second stage, the bearing temperature oscillates between $90 \mathrm{C}$ and $97 \mathrm{C}$.

Analysis of the coupling proves that its temperature is less changeable during operation than the temperature of the bearing. This range is in the range $56 \mathrm{C}$ to $68 \mathrm{C}$.

On the basis of the obtained test results and technical recommendations of the manufacturer of the tested devices, the procedure of control procedure was developed. It also defines information flow, decision making and report archiving.

The procedure presented in Figure 9 specifies four variants of conduct depending on the value of the bearing and coupling temperature of the device. The range (smallest) of the temperature represented in green area defines the nominal operating conditions of the drive while the highest temperature values (red one) determine the critical condition that requires a decision to stop and repair the device.

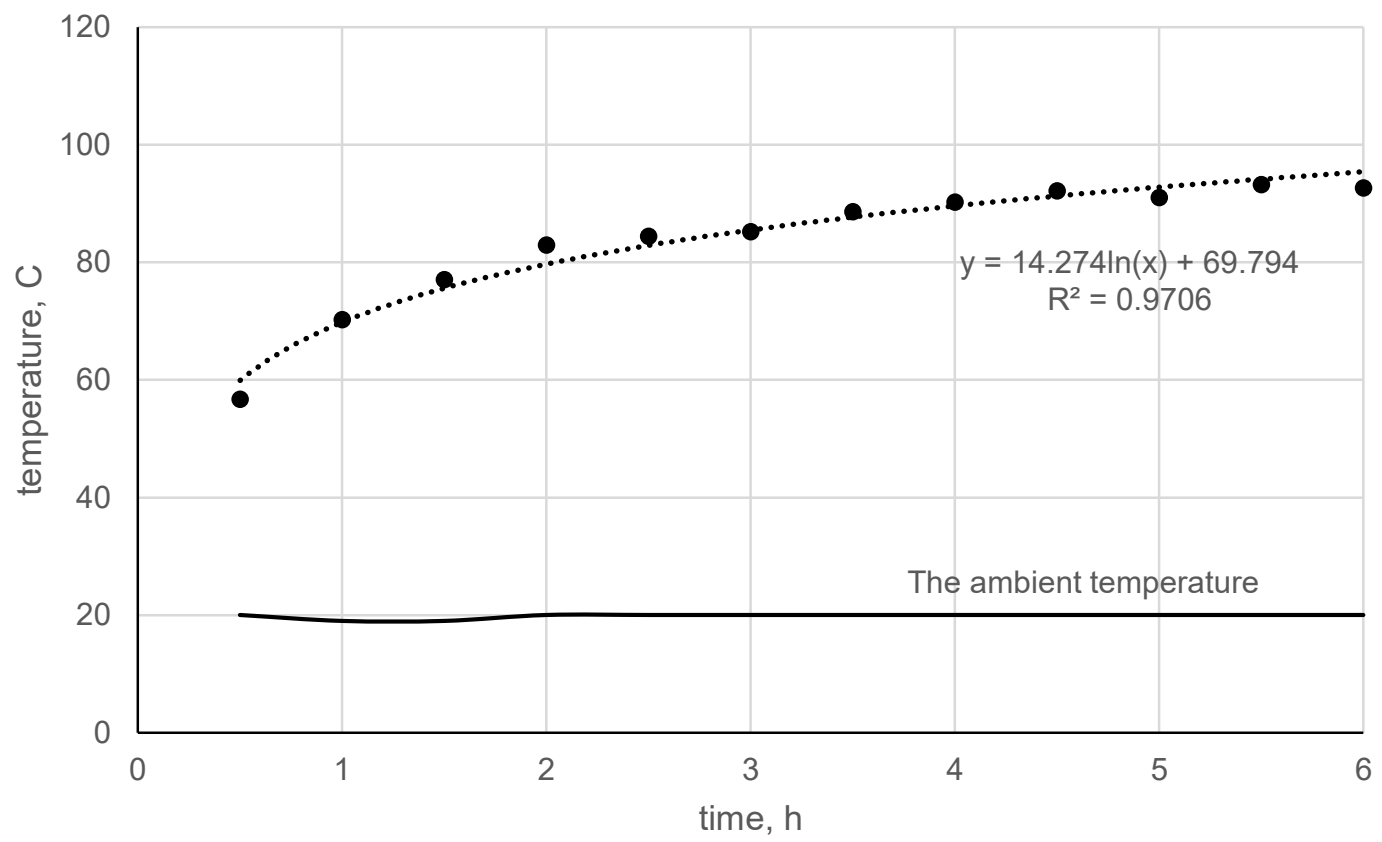

Fig. 5. Time distribution of the temperature of motor bearing of ball mill during the first working shift 
10.21611/qirt.2018.093

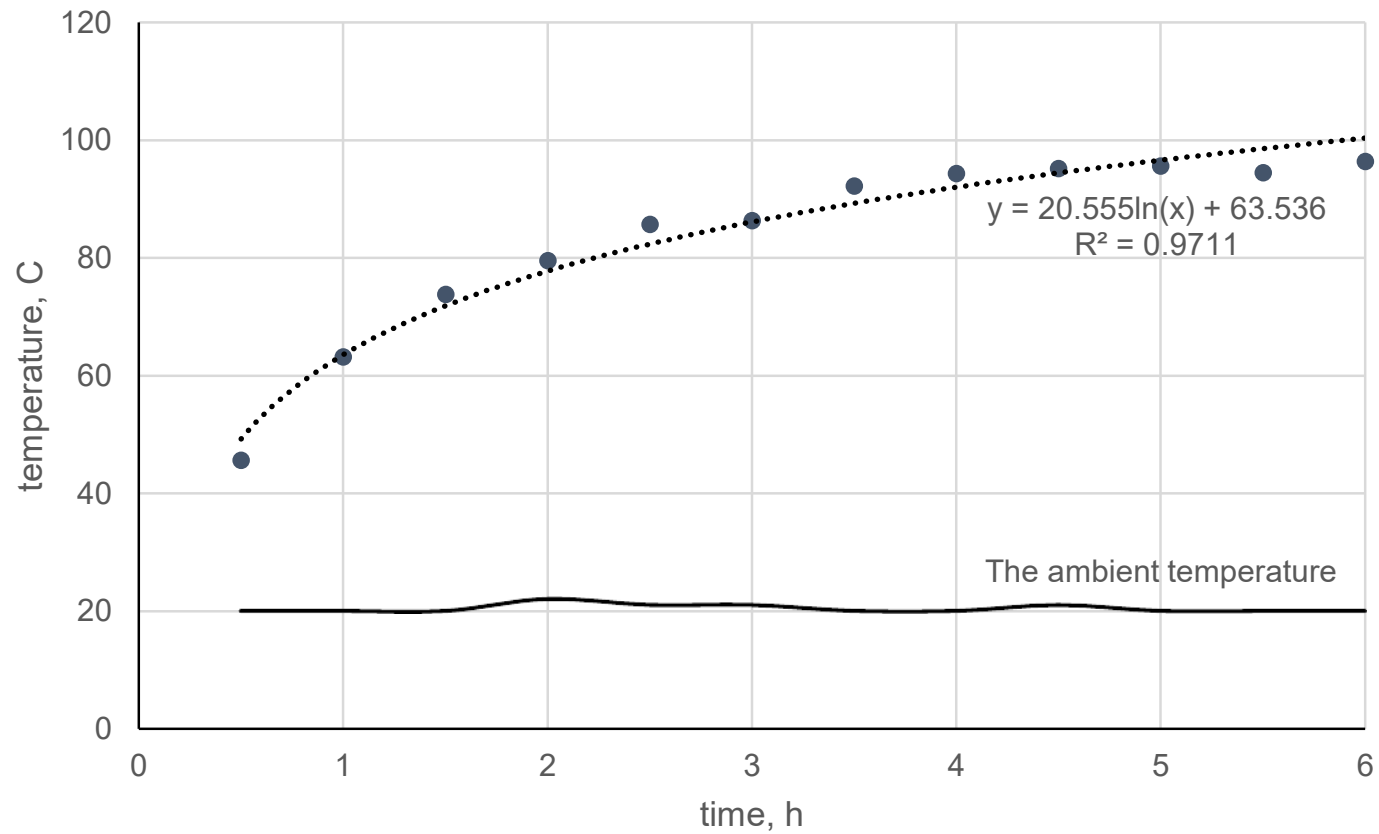

Fig. 6. Time distribution of the temperature of motor bearing of ball mill during the second working shift

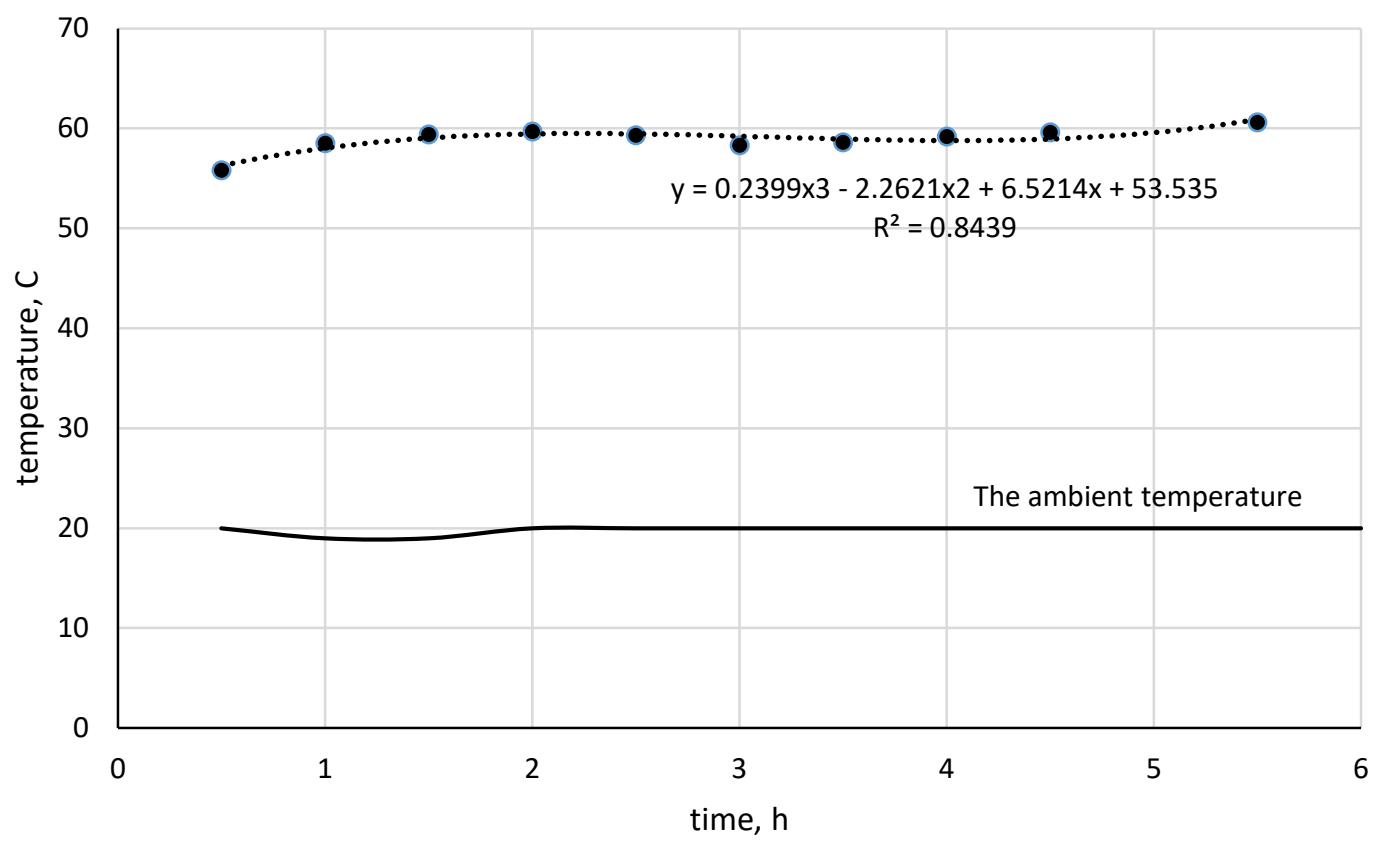

Fig. 7. Time distribution of the temperature of coupling of ball mill during the first working shift 


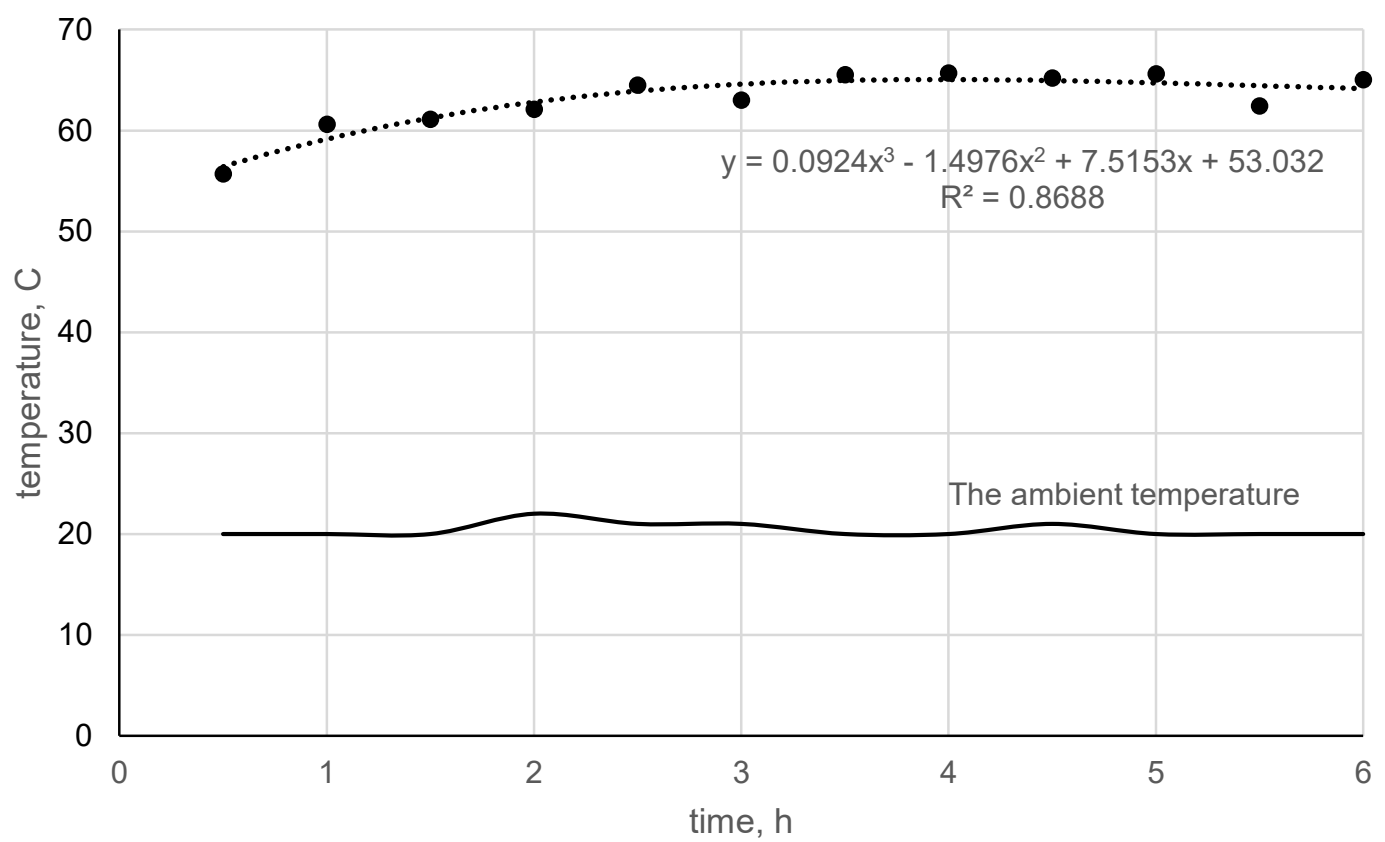

Fig. 8. Time distribution of the temperature of coupling of ball mill during the second working shift

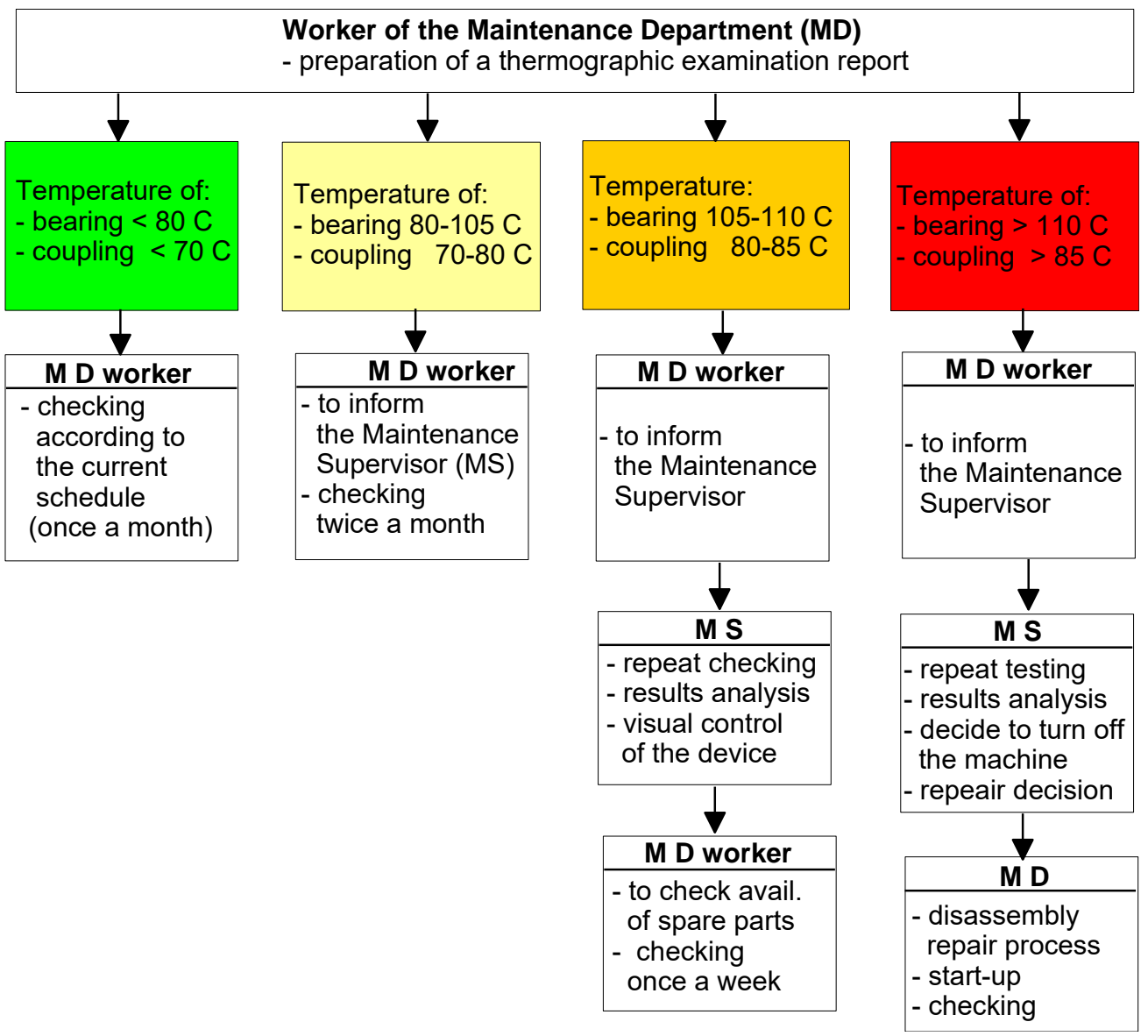

Fig. 9. The procedure of activities related to the test results of temperature distribution of ball mill drive 


\section{Conclusion}

Thermographic tests of electric machines drives are an easy and effective method of monitoring their work, which allows forecasting durability and preventing failures. These studies also indicate the existence of large differences in the temperature distribution not only in the course of a single test, but also in comparing different series of measurements of the same device.

The conducted research shows how much influence on the measurement result has the surrounding - humidity, ambient temperature, dustiness of the surrounding air and emissivity of the surface of the tested object. The last of these factors significantly affects the final result. The emissivity of the housing of the test drive was set at 0.81 . One of the measurements with this parameter indicated the maximum bearing temperature of $95.2^{\circ} \mathrm{C}$.

Not enough careful determination of the emissivity of the tested object can be caused by, for example, surface contamination or difficult access to machines through the shield. Such operation may reduce the value of the indicated temperature, leading the camera operator to disregard the critical state and consequently damage the device. Therefore, it is very important to precisely determine the measurement parameters included in the standard control and measurement procedure.

\section{REFERENCES}

[1] Gade, R.; Moeslund, T.B. Thermal cameras and applications: A survey. Mach. Vision Appl. 25, 245-262, 2014

[2] Więcek B., De May G.: Thermovision in Infrared Radiation Basics and Applications. Warszawa, 2011.

[3] X. Maldague: Applications of infrared thermography in nondestructive evaluation, 2000

[4] Usamentiaga R., Venegas P., Guerediaga P, Vega L., Molleda J., Bulnes F.G.: Infrared Thermography for Temperature Measurement and Non-Destructive Testing. Sensors, 14(7), 12305-12348,2014

[5] Rogler, R.D., Lobl, H., Schmidt, J.: A diagnostic system for live electrical joints in power transmission systems. In: Forty-Second IEEE Holm Conference on Electrical Contacts. Joint with the 18th International Conference on Electrical Contacts, 1996

[6] Jadin, M.S., Ghazali, K.H., Taib, S.: Thermal condition monitoring of electrical installations based on infrared image analysis. In: Saudi International Electronics, Communications and Photonics Conference, 2013

[7] R. Władysiak, A. Kozuń, T. Pacyniak: Examining the Possibilities of Analyzing the Solidification Process of Al-Si Alloy with the Infrared Camera. Archives of Foudry Engineering, vol 16 (1), 89-94, 2016

[8] Kozłowska J.: The use of the thermal imaging camera in the monitoring of electric drived devices in the production. Unpublished M.Sc. dissertation. Lodz University of Technology, 2017

[9] Mobley R.M: An Introduction to Predictive Maintenance. Utterworth-Heinemann, 2002 